\title{
Core-Shell Electrospun Fibers with An Improved Open Pore Structure for Size-Controlled Delivery of Nanoparticles
}

\author{
Zahra Mahdieh¹, Somenath Mitra², Andrij Holian ${ }^{3^{*}}$
}

1- Materials Science Program, Department of Biomedical and Pharmaceutical Sciences, University of Montana, 32 Campus Drive, Missoula, MT, 59812, USA. zahra.mahdieh@umontana.edu

2- Department of Chemistry and Environmental Science, New Jersey Institute of Technology, 323 Dr M.L.K. Jr. Blvd, Newark, NJ 07102, USA. Somenath.mitra@njit.edu

3- *Corresponding author: Center for Environmental Health Sciences, Department of Biomedical and Pharmaceutical Sciences, University of Montana, 32 Campus Drive, Missoula, MT, 59812, USA. andrij.holian@umontana.edu

\section{Supporting Information}

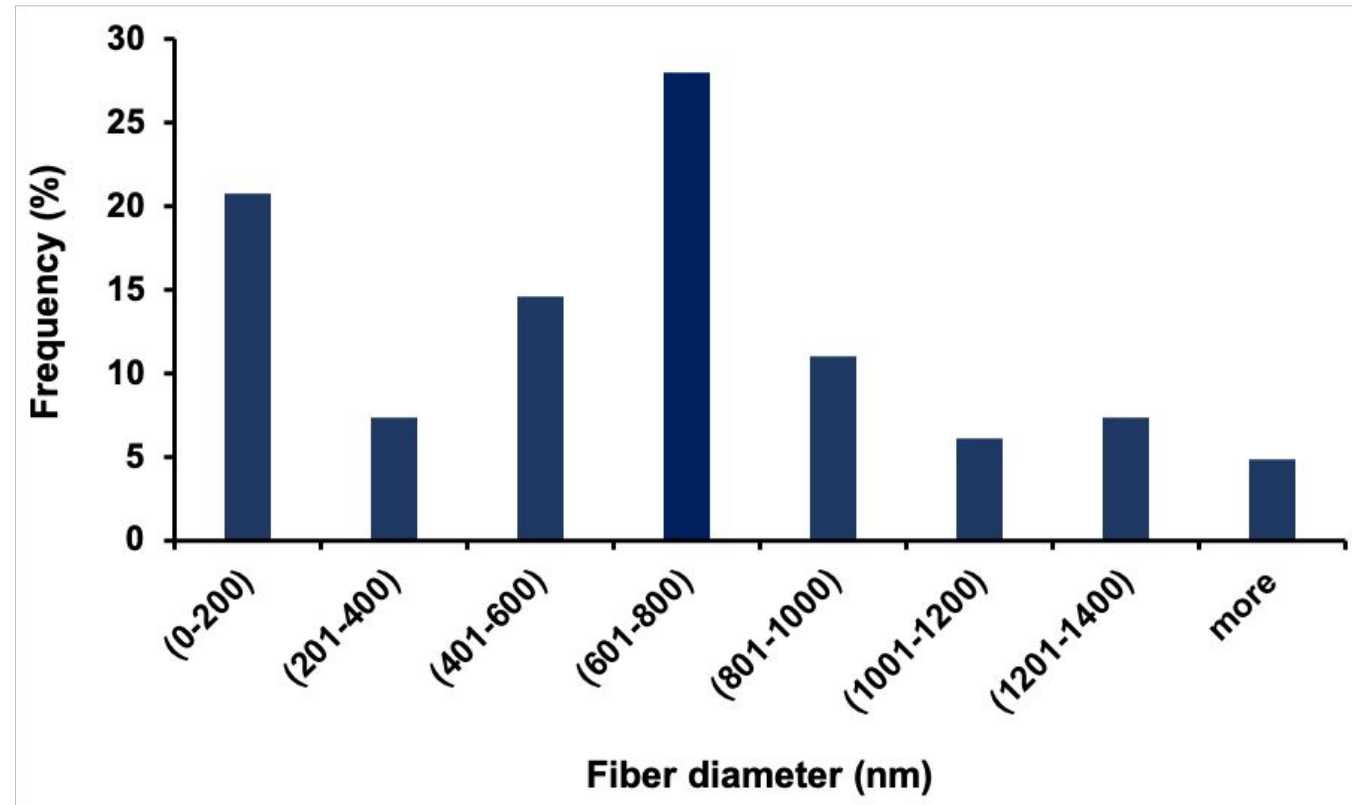

Figure S1. Fiber diameter distribution of fiber mesh F-Ag20 before pore formation. F-Ag20 is the core-shell structured electrospun fiber mesh containing $20 \mathrm{~nm}$ Ag NP in the core. 


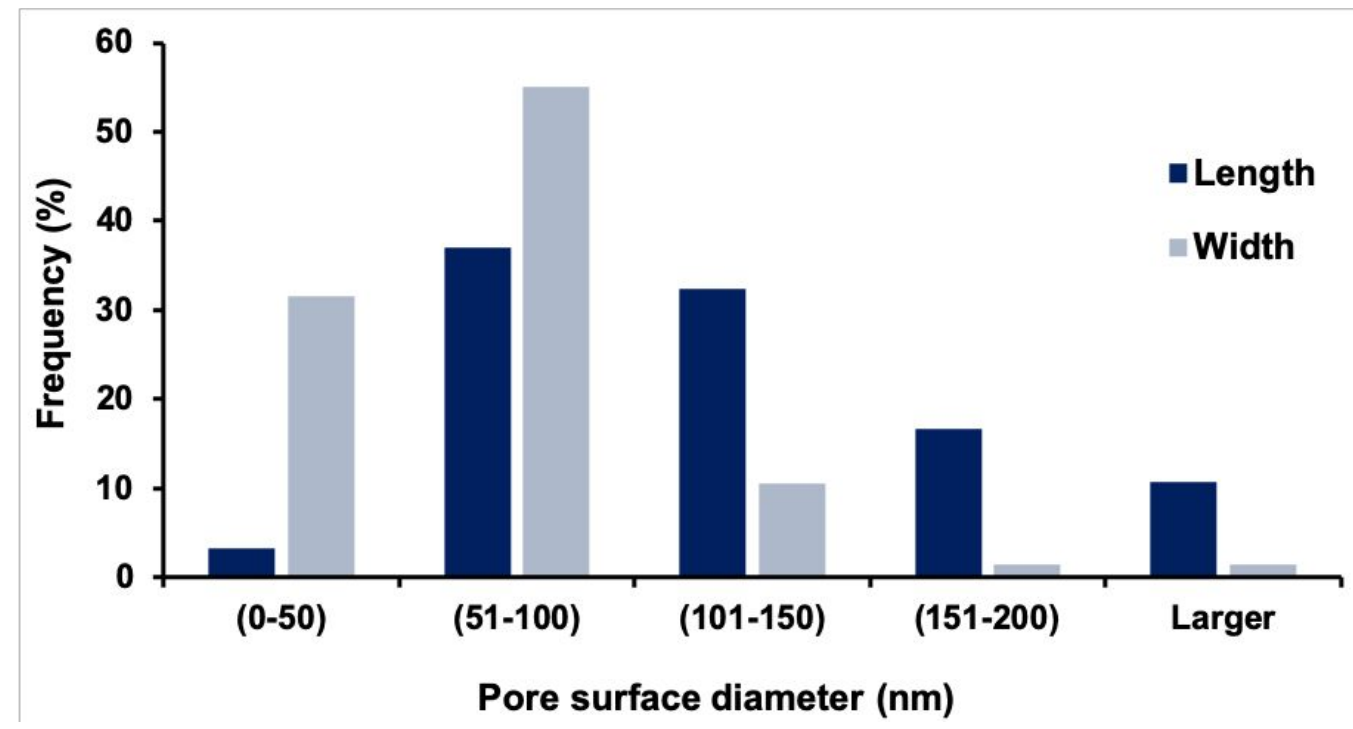

Figure S2. The length and width distribution of the pores formed on the fiber mesh F-Ag20 after one-week immersion measured from FESEM micrographs using ImageJ software. F-Ag20 is the core-shell structured electrospun fiber mesh containing $20 \mathrm{~nm} \mathrm{Ag} \mathrm{NP}$ in the core.

Table S1. BET surface area and pore volume measurements of fiber mesh F-Ag20 before pore formation and after pore formation (one-week immersion). F-Ag20 is the core-shell structured electrospun fiber mesh containing $20 \mathrm{~nm}$ Ag NP in the core.

\begin{tabular}{|l|l|l|}
\hline & Before pore formation & After pore formation \\
\hline Surface area $\left(\mathbf{m}^{2} / \mathbf{g}\right)$ & 29.4 & 173 \\
\hline Pore volume $\left(\mathbf{c m}^{3} / \mathbf{g}\right)$ & 0.012 & 0.076 \\
\hline
\end{tabular}


Table S2. The Holm-Sidak's multiple comparisons test results for the percent cumulative release of $\mathrm{Ag}$ from fiber meshes reported in Figure 8 of the manuscript. Asterisks indicate significant difference ${ }^{* * *} p<0.0001,{ }^{* * *} p<0.0002,{ }^{* *} p<0.0021,{ }^{*} p<0.0332,{ }^{n s} P>0.1234$.

\begin{tabular}{|c|c|c|c|}
\hline & F-Ag20 vs. F-AgMix & F-Ag20 vs. F-Ag110 & F-AgMix vs. F-Ag110 \\
\hline $1 \mathrm{~h}$ & ns & ns & * \\
\hline $2 \mathrm{~h}$ & ns & ns & ** \\
\hline $4 h$ & * & ns & $* * *$ \\
\hline $8 \mathrm{~h}$ & $\star \star \star *$ & ns & $\star * * *$ \\
\hline $24 \mathrm{~h}$ & $\star \star \star \star * \star$ & ns & $\star * * * *$ \\
\hline $72 \mathrm{~h}$ & $\star \star * *$ & ns & $\star \star * * *$ \\
\hline 168h & **** & $* * *$ & 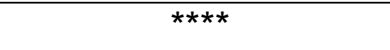 \\
\hline $336 \mathrm{~h}$ & 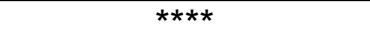 & 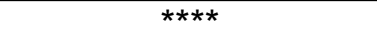 & $\star \star \star * \star *$ \\
\hline
\end{tabular}

Table S3. The Holm-Sidak's multiple comparisons test results for the percent cumulative release of $\mathrm{Zn}$ from fiber meshes reported in Figure 9 of the manuscript. Asterisks indicate significant difference ${ }^{* * *} p<0.0001,{ }^{* * *} p<0.0002,{ }^{* *} p<0.0021,{ }^{*} p<0.0332,{ }^{n s} P>0.1234$.

\begin{tabular}{|l|c|c|c|}
\hline & F-Ag20 vs. F-AgMix & F-Ag20 vs. F-Ag110 & F-AgMix vs. F-Ag110 \\
\hline $\mathbf{1 h}$ & $\mathrm{ns}$ & $\mathrm{ns}$ & $\mathrm{ns}$ \\
\hline $\mathbf{2 h}$ & $\mathrm{ns}$ & $\mathrm{ns}$ & $\mathrm{ns}$ \\
\hline $\mathbf{4 h}$ & $\mathrm{ns}$ & $\mathrm{ns}$ & $\mathrm{ns}$ \\
\hline $\mathbf{8 h}$ & $\mathrm{ns}$ & $\mathrm{ns}$ & $\mathrm{ns}$ \\
\hline $\mathbf{2 4 h}$ & $\mathrm{ns}$ & $\mathrm{ns}$ & $\mathrm{ns}$ \\
\hline $\mathbf{7 2 h}$ & $\mathrm{ns}$ & $\mathrm{ns}$ & \multirow{2}{*}{} \\
\hline $\mathbf{1 6 8 h}$ & $\mathrm{ns}$ & $\mathrm{ns}$ & \multirow{2}{****}{} \\
\hline $\mathbf{3 3 6 h}$ & $\mathrm{ns}$ & & \\
\hline
\end{tabular}


<smiles>CCCCCCC(=O)OCCCCCCC(=O)OCCCCCCC(=O)OC</smiles>

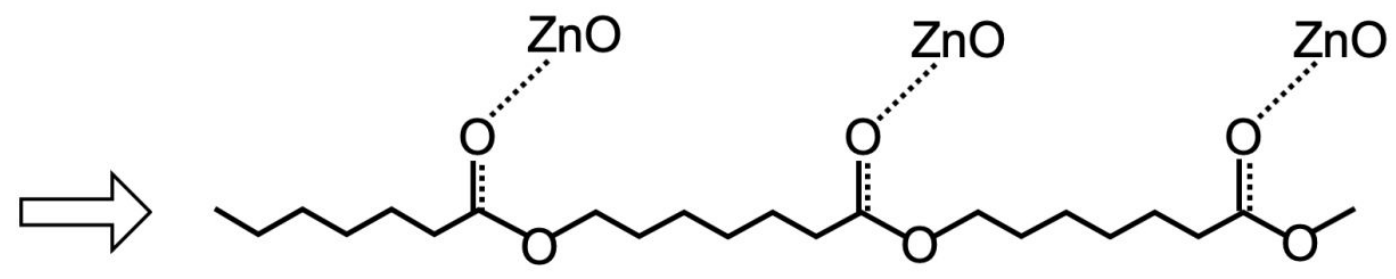

Figure S3. Schematic presentation of ZnO NP and PCL polymer chain interaction. Reproduced with permission from reference (63).

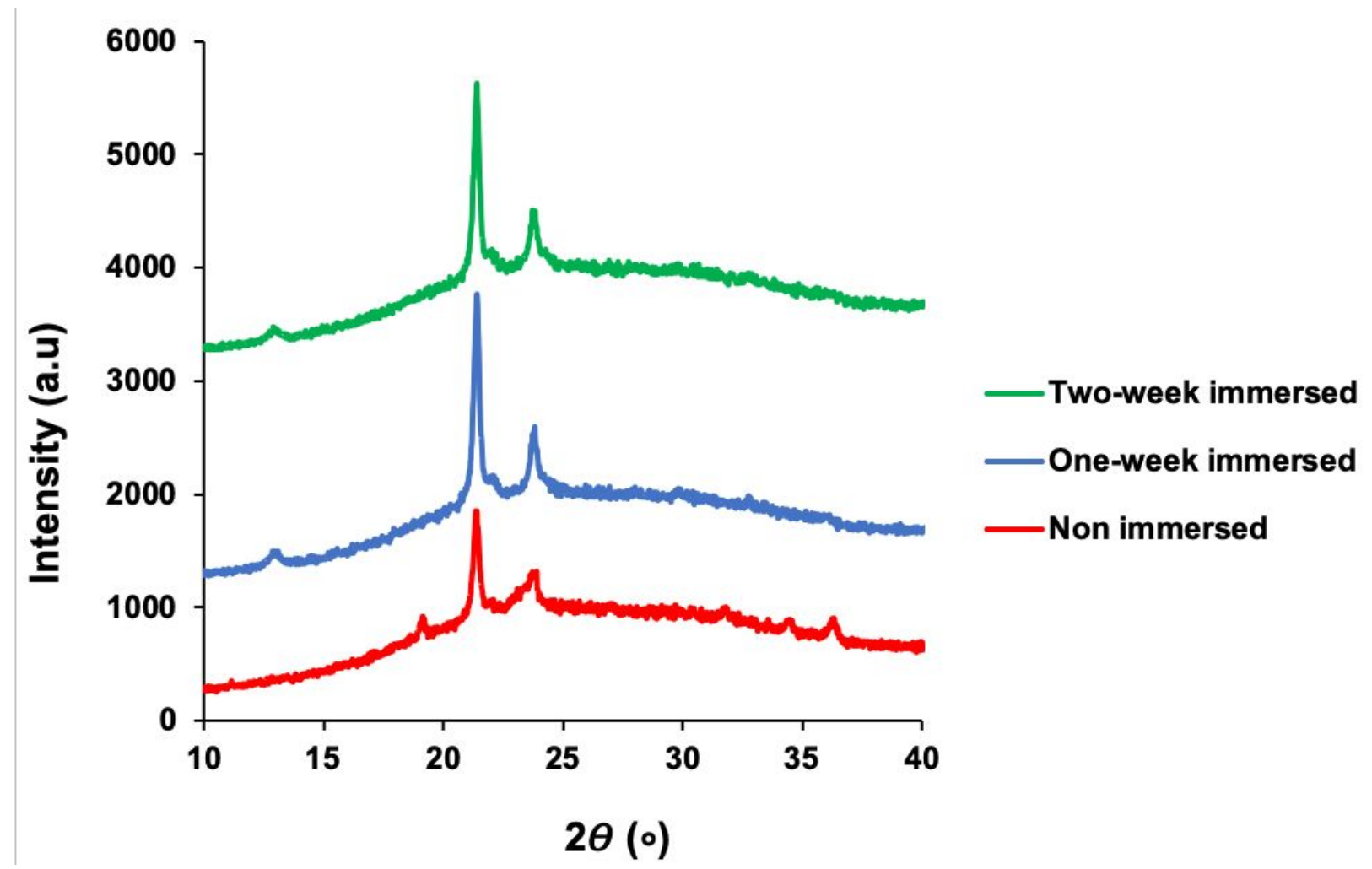

Figure S4. XRD diffractograms of fiber mesh F-Ag20: before immersion, one-week immersed, and two-week immersed. F-Ag20 is the core-shell structured electrospun fiber mesh containing $20 \mathrm{~nm}$ Ag NP in the core. Diffractograms are separated from one another to show the peaks distinctly. 
Table S4. The Holm-Sidak's multiple comparisons test results for the measured mechanical properties (elongation at break, ultimate tensile strength, and Young's modulus) of non-, one-, and two-week immersed fiber mesh F-Ag20 reported in Table 3 of the manuscript. Asterisks indicate significant difference ${ }^{* * * *} p<0.0001,{ }^{* * *} p<0.0002,{ }^{* *} p<0.0021,{ }^{*} p<0.0332$, ns $P>$ 0.1234 .

\begin{tabular}{|l|c|c|c|}
\hline & $\begin{array}{l}\text { Non-immersed vs. } \\
\text { one-week immersed }\end{array}$ & $\begin{array}{l}\text { Non-immersed vs. } \\
\text { two-week immersed }\end{array}$ & $\begin{array}{l}\text { one-week immersed vs. } \\
\text { two-week immersed }\end{array}$ \\
\hline Elongation at break & $* *$ & ${ }^{* *}$ & $\mathrm{~ns}$ \\
\hline Ultimate tensile strength & $* * *$ & $\mathrm{~ns}$ & ${ }^{*}$ \\
\hline Young's modulus & $\mathrm{ns}$ & ${ }^{*}$ & \\
\hline
\end{tabular}

Table S5. Measured tensile properties (elongation at break, ultimate tensile strength, and Young's modulus) of non-, one-, and two-week immersed fiber mesh F-Ag20. The SD for n=4 is reported in the parentheses for each measurement. F-Ag20 is the core-shell structured electrospun fiber mesh containing $20 \mathrm{~nm}$ Ag NP in the core.

\begin{tabular}{|l|l|l|l|}
\hline & $\begin{array}{l}\text { Elongation at } \\
\text { Break (\%) }\end{array}$ & $\begin{array}{l}\text { Ultimate Tensile } \\
\text { Strength (KPa) }\end{array}$ & $\begin{array}{l}\text { Young's } \\
\text { Modulus (KPa) }\end{array}$ \\
\hline Non-immersed & $41.33( \pm 6.58)$ & $919.46( \pm 72.99)$ & $88.56( \pm 1.62)$ \\
\hline $\begin{array}{l}\text { One-week } \\
\text { immersed }\end{array}$ & $89.10( \pm 12.36)$ & $\begin{array}{l}1466.55 \\
( \pm 123.23)\end{array}$ & $83.40( \pm 18.52)$ \\
\hline $\begin{array}{l}\text { Two-week } \\
\text { immersed }\end{array}$ & $72.79( \pm 4.11)$ & $1067.60( \pm 53.77)$ & $49.37( \pm 2.43)$ \\
\hline
\end{tabular}

\section{Supporting information references:}

(63) Augustine R, Malik HN, Singhal DK, Mukherjee A, Malakar D, Kalarikkal N, Thomas S. Electrospun polycaprolactone/ZnO nanocomposite membranes as biomaterials with antibacterial and cell adhesion properties. J Polym Res. 2014 Mar 13;21(3):347. 